\title{
Conductivity and Electroosmotic Transport in Nanofluidic Channels
}

\author{
Daniel G. Haywood, Zachary D. Harms, Stephen C. Jacobson \\ Department of Chemistry, Indiana University, Bloomington, IN 47405 USA
}

Ion transport in nanofluidic devices has received substantial attention due to unique properties exhibited at these length scales. Phenomena such as double layer overlap and surface conductivity that are insignificant in microchannels can impact transport properties in nanochannels [1]. Here, we describe the effects that nanometer dimensions have on transport in glass nanofluidic devices i.e., increased conductivity of nanochannels due to surface charge and the reduction of electroosmotic velocities at low buffer concentrations due to double-layer overlap.

Devices consisting of two V-shaped microchannels were fabricated in borosilicate glass substrates by UV photolithography and wet chemical etching. A focused ion beam (FIB) instrument was used to mill straight nanochannels of varying depths into the glass substrate to bridge the $20-\mu \mathrm{m}$ gap between the microchannels. After milling, the substrates were irreversibly bonded to a cover plate by thermal annealing. A schematic of the device and a scanning electron microscope image of a $100-\mathrm{nm} \times 500-\mathrm{nm}$ nanochannel are shown in Figure 1.

Conductivities in 50,100, and 200-nm straight nanochannels were measured over a range of electrolyte concentrations and compared to the solution conductivities. Figure 2 shows that at high electrolyte concentrations the nanochannel conductivities were the same as the bulk conductivities. However, at low electrolyte concentrations, the conductivity observed in the nanochannels is greater than expected. Conductivities in the nanochannels level off at low $\mathrm{NaCl}$ concentrations due to the presence of surface charge and the high surface-to-volume ratio [2].

Impact of the channel dimensions on electroosmotic flow was studied by measuring the velocity of a zwitterionic dye. A potential was applied across the nanochannel to determine its arrival time at a point along the channel. Velocities at varying $\mathrm{NaCl}$ concentrations at different field strengths are plotted in Figure 3. A maximum velocity was observed at $10 \mathrm{mM} \mathrm{NaCl}$, which, interestingly, is the same concentration where the conductivity begins to deviate from the bulk. Further reduction in the electroosmotic mobility at $1 \mathrm{mM} \mathrm{NaCl}$ is shown in Figure 4, and is caused by double-layer overlap in the nanochannel [3]. 
References:

[1] C.L. Rice, R. Whitehead, Journal of Physical Chemistry 69 (1965) 417-424.

[2] D. Stein et al, Physical Review Letters 93 (2004) 35901

[3] This work was supported in part by NSF CHE-0832651, NSF CHE-0923064, and NIH R01 GM100071

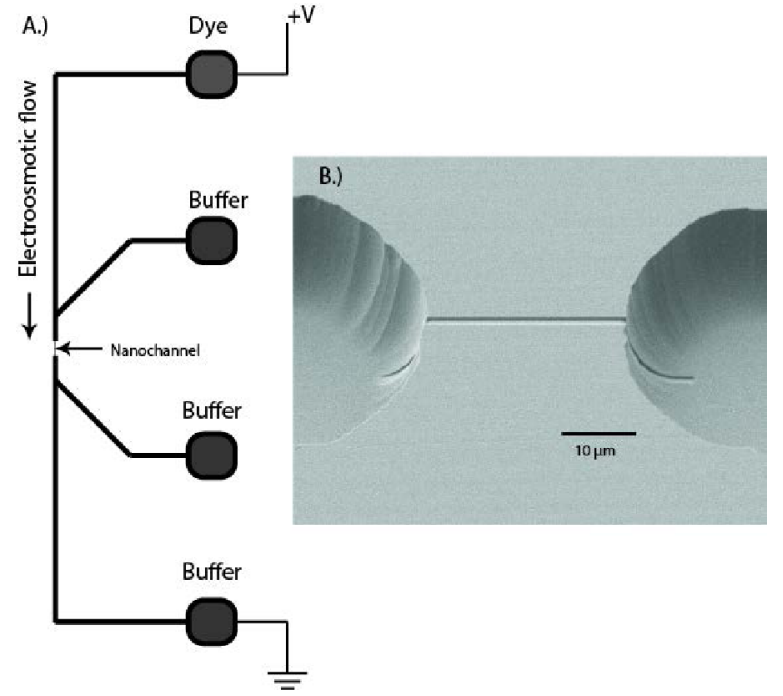

Figure 1. A.) Schematic of nanofluidic device used for conductivity and electroosmotic studies B.) SEM image of FIBmilled nanochannel spanning two microchannels.

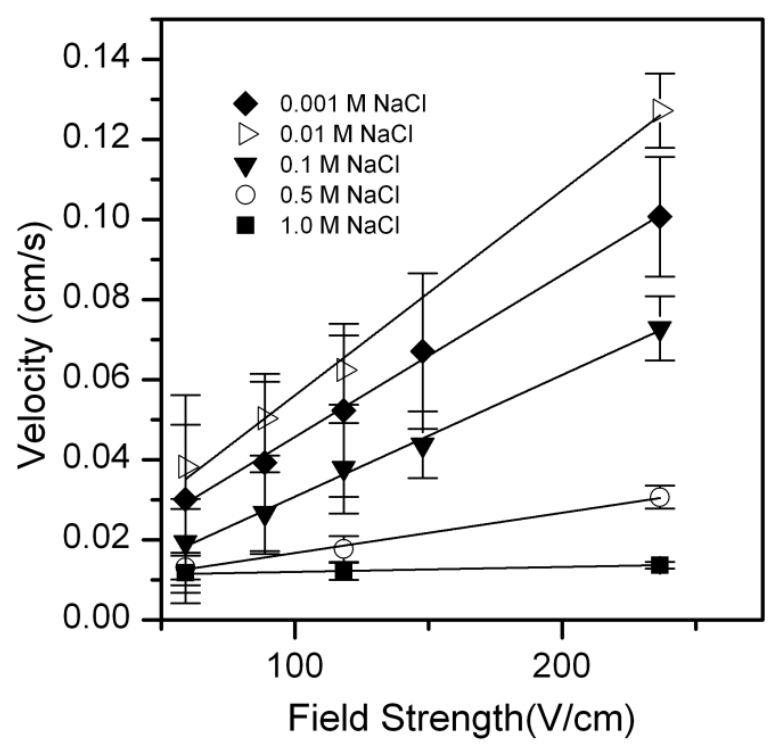

Figure 3. Variation of electroosmotic velocity with field strength for $\mathrm{NaCl}$ solutions from $1 \mathrm{mM}$ to $1 \mathrm{M}$.

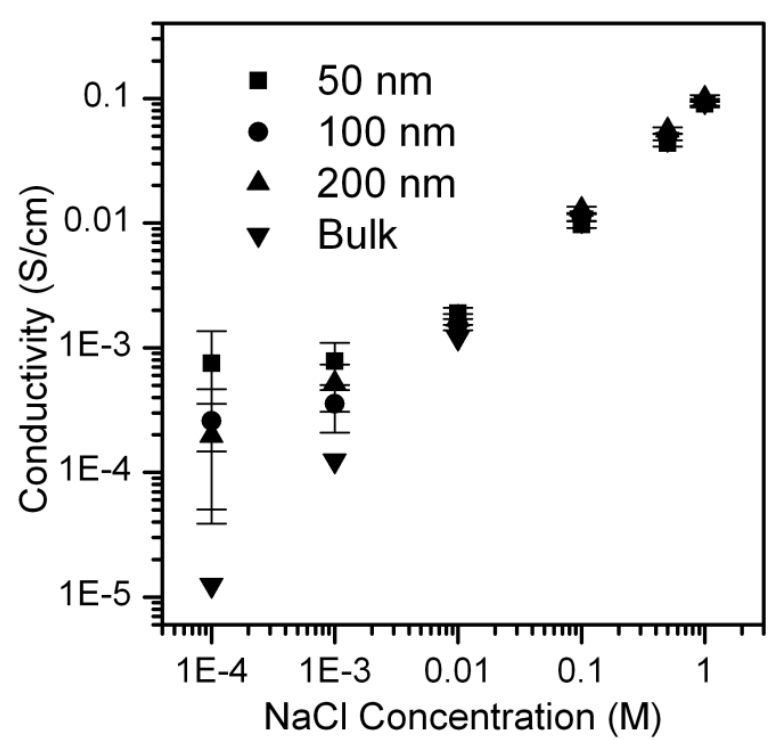

Figure 2. Variation of conductivities with $\mathrm{NaCl}$ concentration for nanochannel depths of 50, 100, 200nm and bulk solutions.

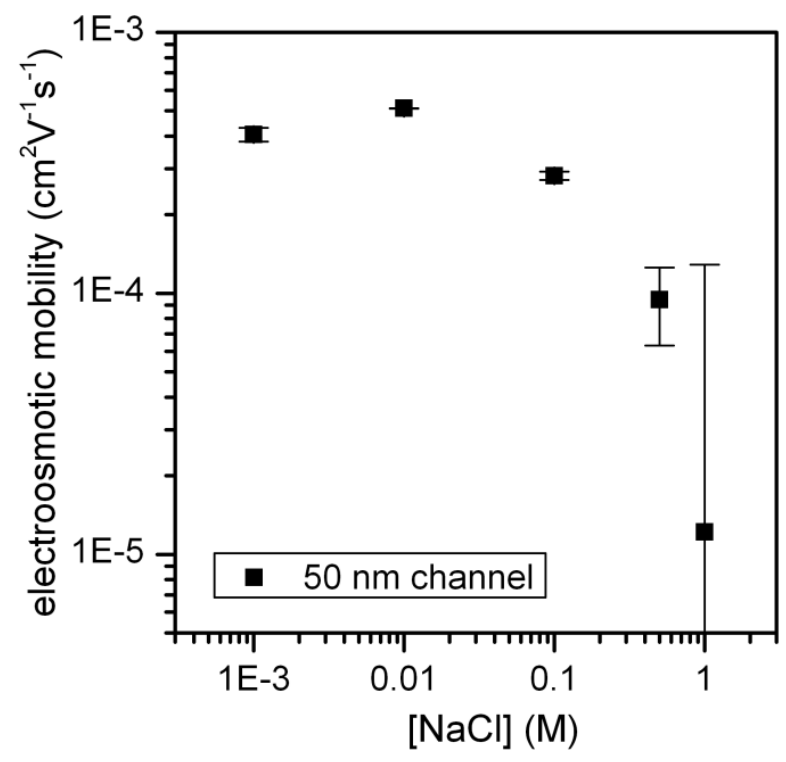

Figure 4. Variation of electroosmotic mobility with $\mathrm{NaCl}$ concentration in a 50-nm deep nanochannel in a glass substrate. 